\title{
Models for Multicomponent Fuzzy Evaluation, with a Focus on the Assessment of Higher-Order Thinking Skills
}

\author{
Emil Hadzhikolev ${ }^{1}$, Stanka Hadzhikoleva ${ }^{2}$, Kostadin Yotov ${ }^{3}$, Daniela Orozova ${ }^{4}$ \\ ${ }^{1,2,3}$ Plovdiv University Paisii Hilendarski, Faculty of Mathematics and Informatics, \\ 236 Bulgaria Bul., Plovdiv, Bulgaria \\ ${ }^{4}$ Burgas Free University, Faculty of Computer Science and Engineering, \\ 62, San Stefano Str., Burgas, Bulgaria \\ ${ }^{4}$ Bulgarian Academy of Sciences, Institute of Information and Communication Technologies, \\ 1, 15 Noemvri Str., Sofia, Bulgaria
}

\begin{abstract}
The increased need to use distance and online forms of training in the modern world forces teachers to seek, develop and use new and diverse teaching models. The pursuit of adequate fair assessment of students' knowledge and skills, their active participation in the learning process, their independent work on projects and case studies has also posed new challenges for teachers. The article presents models for hierarchical multicomponent assessment of students, aimed at comprehensive assessment of various thinking skills of high and low order, theoretical knowledge and practical skills, etc. A fuzzy evaluation approach is used to formalize the assessor's subjective logic.
\end{abstract}

Keywords - fuzzy evaluation, evaluation models, HOTS evaluation, multicomponent assessment.

\section{Introduction}

Education is a complex process that requires consistent acquisition of knowledge and skills, done in an appropriate sequence.

DOI: $10.18421 /$ TEM94-43

https://doi.org/10.18421/TEM94-43

Corresponding author: Stanka Hadzhikoleva, Plovdiv University Paisii Hilendarski, Faculty of Mathematics and Informatics, 236 Bulgaria Bul., Plovdiv, Bulgaria.

Email: stankah@uni-plovdiv.bg

Received: 09 September 2020.

Revised: 25 September 2020.

Accepted: 11 October 2020.

Published: 27 November 2020.

(c) BY-NC-ND C 2020 Emil Hadzhikolev at al; published by UIKTEN. This work is licensed under the Creative Commons Attribution-NonCommercial-NoDerivs 4.0 License.

The article is published with Open Access at www.temjournal.com
For optimal results, the education must be managed by an experienced teacher. Many pedagogical approaches, methods and methodologies on this topic are described in the scientific literature.

One of the notable taxonomies that stood the test of time is Bloom's Taxonomy [1]. It defines a hierarchy of thinking skills in which the higher levels of thinking incorporate all cognitive skills from the lower levels. The levels are structured as follows Knowledge, Comprehension, Application, Analysis, Synthesis, and Evaluation. Each level is determined by multiple cognitive skills, activities and assessment methods. It is believed that in the learning process, a learner goes through all levels successively.

Bloom's Taxonomy is widely used in education. It provides a framework for defining and grading the goals and objectives of the education, planning the learning process activities, formulating problems and setting tasks for learners, choosing assessment tools adequate to the set goals, etc. [2]. A generally accepted classification defines the skills of the top three levels of Bloom's Taxonomy (Analysis, Synthesis, and Evaluation) as higher-order thinking skills (HOTS), and those of the lower three levels (Knowledge, Comprehension, and Application) - as lower-order thinking skills (LOTS). HOTS are extremely valuable and very important for the progress of every member of society, as well as the progress of society as a whole. This includes critical thinking skills, abilities for knowledge and skills transfer, problem solving skills, etc. [3].

The assessment of student's knowledge and skills must be objective, i.e. to make the same assessments in different inspections, regardless of where the assessment takes place and who the assessors are. In many cases this is difficult to achieve, especially when it comes to assessing HOTS that require creative thinking. This is what has motivated our work directed at creating complex multicomponent models for assessing students' achievements. The 
models described in the article draw ideas from the fuzzy set theory. It is a formal apparatus for describing and analysis of complex objects and phenomena in the conditions of fuzzy information. This theory provides a scheme for solving problems in which the subjective assessment plays a significant role in accounting for uncertainty factors. The main assumption of the theory is that the degree of possession of a subjective property by an object is not amenable to objective assessment, or assessment of the type "yes"/"no", but allows a subjective interpretation of a more general nature [4].

\section{Assessment Approaches}

Different forms and methods of testing are used to assess learners' knowledge, such as online or offline tests, answers to open-ended questions, testing and reading of code, solving problems and case studies, work on projects, essays, etc. The final assessment must be complex and involve different components having different weights in its formation.

A detailed review of literature referring to various techniques for multicriteria decision making and their applications is available in [5]. Depending on the problem studied, the assessment criteria can be expressed through a quantitative, qualitative, fuzzy or mixed model. In addition to the assessment criteria, the weights of the criteria can also be expressed in fuzzy numbers or fuzzy ratios to express the meaning of the criteria [6]. There are different classifications of approaches for assessing learners' knowledge depending on the purpose. Problems related to predictive modeling for recruiting and retaining student is presented in [7]. Experiments related to the development of forecasting models based on student admission data are presented in [8], [9]. The idea of multi-criteria models in cluster design could be applied in the ranking and distribution of students in groups for assessment purposes [10].

A model can be applied to assess the work of the learners, taking into account the acquired theoretical knowledge and practical skills separately [11]:

$$
\begin{gathered}
\max _{i=1 . . S}\left(\alpha \sum_{j=1}^{N} w_{j} e_{i j}+\beta \sum_{k=1}^{M} w_{k} e_{i k}\right), \\
\sum_{j=1}^{N} w_{j}=1, \quad \sum_{k=1}^{M} w_{k}=1, \quad \alpha+\beta=1,
\end{gathered}
$$

where $w_{j}$ and $w_{k}$ are relative weighting factors for the measured theoretical $\left(e_{i j}\right)$ and practical $\left(e_{i k}\right)$ criteria of the $i$-th student, and $\alpha$ and $\beta$ show the weights with which theoretical knowledge and practical skills participate in the final assessment.

Approaches for dynamic assessment of learners based on intuitionistic fuzzy evaluations are presented in [12], [13]. In [14] intuitionistic fuzzy evaluations are applied for specific assessment of the knowledge of students in mathematics in university courses for e-learning, and in [15] fuzzy grades are formed on the basis of evaluating the student work by several different lecturers.

\section{Models for Hierarchical Multicomponent Evaluation}

In multicomponent evaluation, the final assessment is a function of many assessment components which are measures of different knowledge and skills, both theoretical and practical. The assessment components can be varied - tests, assignments, term papers, case studies, projects, essays, and many more. They may require individual work as well as teamwork. They can be assessed in different ways - teacher assessment, self-assessment, peer assessment, etc., and by means of different assessment scales and systems.

In the simplest case, the components have equal weight and the score is formed as the arithmetic mean of the scores of the components. In many cases, however, assessors consider some assessment components to be more significant and indicative, so they give them higher weight values. These weight values can be determined intuitively, motivated by taxonomy, depending on the specific domain, scientific field, professional field etc. Another possibility is to determine the weights automatically, e.g. to use methods of artificial intelligence which form new assessments based on accumulated data for previously made evaluations. A similar successful experiment with a neural network is described in [16]. In both main approaches, weights are an explicit or implicit manifestation of the assessor's subjective opinion. In the first case, the assessor clearly sets subjective weights. In the second - the neural network automatically determines the weights but they are based on the assessor's subjective judgment presented through input-output data for training neural networks.

The formation of the final assessment depends on the assessor's subjective judgment for some other reasons as well. The subjective factor is most often manifested in borderline cases where the assessor hesitates between two discrete values for the final assessment, when its real value is between the two. For example, for a rating scale from 2 to 6, borderline problem cases are those in which the actual ranges are around 2.5, 3.5, 4.5, 5.5.

Despite the good results for automated assessment obtained with neural networks and other methods of artificial intelligence, in the general case there is no clarity for the objectivity of the assessments made. The assessor may be left with a sense of distrust in the automated evaluation, which has its real grounds. 
The neural network is trained on input-output samples provided by the assessor, and sometimes samples for certain boundary cases may be missing. The neural network creates a function on the input variables which approaches the logic provided by the samples, but not all possible cases. Determining and collecting a sufficient number of input-output data for a neural network in multidimensional spaces is also a complex issue. The algorithmic increase in the number of input-output samples for neural network training is an approach successfully experimented by us and is briefly presented in [16]. Nevertheless, artificial intelligence methods remain based on subjective assessments provided by the assessor.

A possible approach to creating a complex solution is the examination of multiple evaluation methods in order to compare them and possibly use them simultaneously in an automated assessment system.

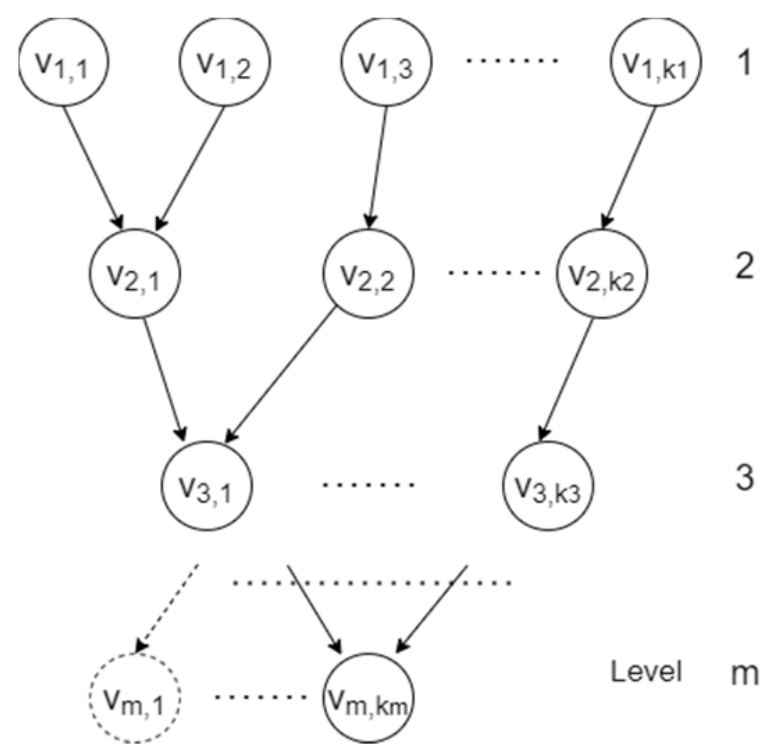

Figure 1.a) Model 1: Hierarchical tree-like organization of assessment components

Figure 1.a) presents a general Model 1 of a hierarchical tree organization of the assessment components. In it, each main component, as well as the final assessment, depends on multiple subcomponents at the previous level. Furthermore, each sub-component affects only one higher-level component.

Model 2 of hierarchical graph organization of assessment components (fig. 2.b) expands and summarizes the capabilities of Model 1. Main extensions are derived from the ability of a single component to affect multiple components from arbitrary higher levels, as well as to depend on components at different lower levels.

The presented models could be used in many different approaches to evaluation where the final assessment is a function of multiple assessment components. Main characteristics of the proposed models are:
Working in this direction, we focused on modeling the hierarchical organization of the assessment components and the dependencies between them which the assessor explicitly or implicitly uses in assessment (fig. 1). For example, in many cources, the main assessment components are "practice" and "theory", and in terms of thinking skills, their respective subcomponents can be theoretical and practical LOTS and HOTS. On the other hand, if we choose HOTS and LOTS as the main components, they could have sub-components for assessing theoretical knowledge and practical skills. A specific hierarchy of components and subcomponents can be created using pre-standardized models selected by the assessor.

It should be noted here that with sufficient inputoutput samples and effective neural network training, the results obtained should reflect this hierarchical organization even if it is not explicitly indicated.

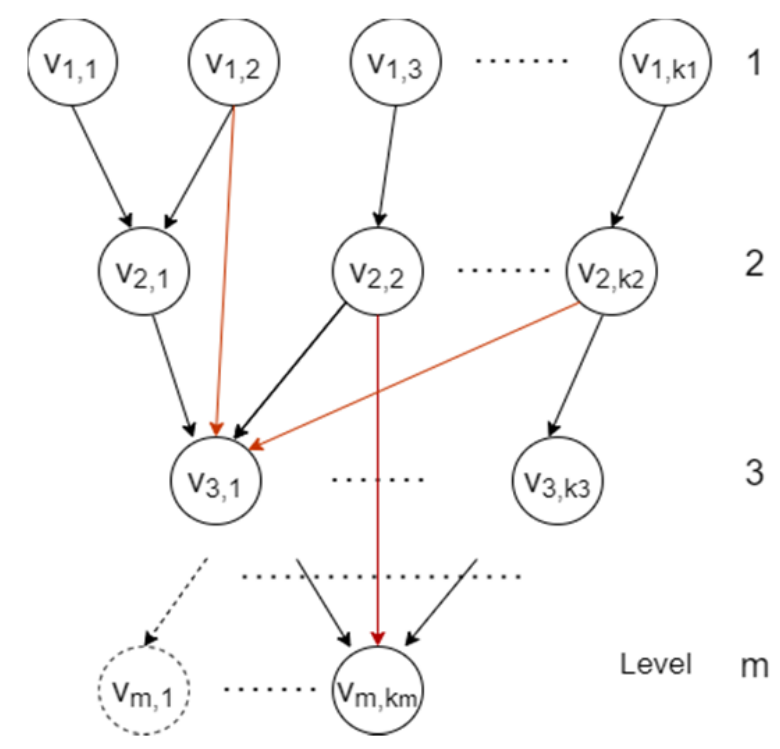

Figure 1.b) Model 2: Hierarchical graph organization of assessment components

- Level 1 of the component hierarchy describes specific values from conducted evaluations which can have different assessment scales, incl. written or oral, practical or theoretical. Examples of such values are: scores for theory and practice tests; scores for skill test according to Bloom's Taxonomy; assessment of assignments, course projects, essays, case studies, etc.

- At each subsequent level, the components form an assessment which is a function of the assessments of its subordinate sub-components from the previous level. In particular, all or part of the functions may have fuzzy logic.

- At the last level there is one component, i.e. $k_{m}=1$, but this is not mandatory. There may be several final assessments, e.g. for different evaluated cognitive skills. In that $k_{m} \geq 1$. 
A possible option for level 2 functions is the rationing of all values of the level 1 components to a common evaluation system (e.g. from 2 to 6). Similarly, the functions at each subsequent level could maintain the rationing of the parent components in the respective evaluation scale. The reason for such an approach to reasoning is the adherence to the logic of the assessor who often thinks in the categories of a specific evaluation scale. Of course, such an approach is not mandatory, and the general model allows for any intermediate results. The final assessment could, for example, be cumulative and get formed as the sum of the subcomponents.

Usually, the evaluation sub-components have values in the space of real positive numbers $R^{+}$. In different evaluation approaches, the values can be in other spaces of real, complex or other numbers. The final grade $E$ however, must belong to a space of predetermined possible values - e.g. it must be an integer in the range from 2 to 6 , from 1 to 5 , from $\mathrm{A}$ to $\mathrm{F}$, etc.

In the formal mathematical description of Model 1 and Model 2, each level with a number $i$ has $k_{i}$ components defining the set:

(1) $V_{i}=\left(v_{i, 1}, v_{i, 2}, \ldots, v_{i, k_{i}}\right) \in R^{k_{i}}, k_{i} \in N, i=1 . . m$

Level 1 components are specific values obtained during evaluation. At each subsequent level $\mathrm{i}=2 . . \mathrm{m}$ the values are obtained as a result of applying functions to the components of the previous level. In Model 1 the number of components in a level decreases or remains equal to the number of components in the previous level $k_{s} \leq k_{s-1}, \mathrm{~s}=$ $2 . . m$, which is a consequence of combining subcomponents into components. The number of summarizing functions, and respectively of the components, can increase in certain levels with Model 2.

In Model 1, the components' values after level 1 are calculated as a function on values of the components of the direct parental levels:

$$
\begin{aligned}
& \text { (2) } v_{i, j}=f_{i, j}\left(P_{i-1, j}\right) \\
& P_{i-1, j}=\left(v_{i-1, s_{1}}, v_{i-1, s_{2}}, \ldots, v_{i-1, s_{j}}\right) \subseteq V_{i-1}, \\
& \forall i=2 . . m, j=1 . . k_{i}, s_{j} \leq k_{i-1} \text {, and the components } \\
& \text { of } V_{i-1} \text { meet only once in any } P_{i-1, j} .
\end{aligned}
$$

In Model 2, parameters of the functions at a given level can be arbitrary assessment components from all previous levels, thus removing many restrictions:

$$
\text { (3) } \begin{aligned}
& v_{i, j}=f_{i, j}(Q), Q \subseteq \bigcup_{r=1}^{i-1} V_{r}, \\
& \forall i=2 . . m, j=1 . . k_{i} .
\end{aligned}
$$

\section{Use of Fuzzy Logic in Hierarchical Multicomponent Evaluation}

In the exams conducted in the discipline "Internet Programming", we assess theoretical knowledge and practical programming skills through four exam components:

- tLOTS - theoretical LOTS, assessed by scores in the range $[0,30]$;

- pLOTS - practical LOTS, the assessment is an integer in the range $[0,15]$;

- tHOTS - theoretical HOTS, the assessment is an integer in the range $[0,15]$;

- pHOTS - practical HOTS, the assessment is a real number in the range $[2,6]$.

The pHOTS assessment is formed with a practical task and belongs to the range $[2,6]$. The scores for the other evaluation components are formed using a test in paper or electronic form.

The final assessment finalN is an integer on the evaluation scale $[2,6]$, i.e. final $\in[2,6] \subset N$. Based on Model 1 of hierarchical tree organization of the evaluation components, we define two main possible options for formalizing the assessor's logic for assessment in the respective discipline (fig. 2). If necessary, by defining additional dependencies between components at different levels, we can easily move to Model 2. Other options could also be created and experimented in different situations and disciplines in practice. What is important in any case is to determine the components' hierarchy and relationship, as well as the set of their respective aggregate functions suitable for a given evaluation.

The main components forming the final assessment in Variant 1 for the theoretical and practical hierarchical multicomponent evaluation are theory and practice (fig. 2.a). Sub-components of the theory are normalized forms of the primary components $t L O T S$ and $t$ HOTS, and in practice - the normalized forms of pLOTS and pHOTS. The purpose of the normalized forms is to reduce the primary components to a uniform evaluation scale (in this case, 2 to 6), so that they can be presented in mental frameworks for assessment that are closer to the assessor's framework.

On the other hand, in Variant 2 for HOTS-LOTS hierarchical multicomponent evaluation (fig. 2.b), HOTS and LOTS are accepted as primary components and their sub-components are respectively the normalized forms of the practical and theoretical HOTS, and the practical and theoretical LOTS.

In both modeled variants normalized forms of the main evaluation components are at the first functional level. Specific normalizing functions can implement a liberal or strict approach for evaluation, or their modification, depending on the 


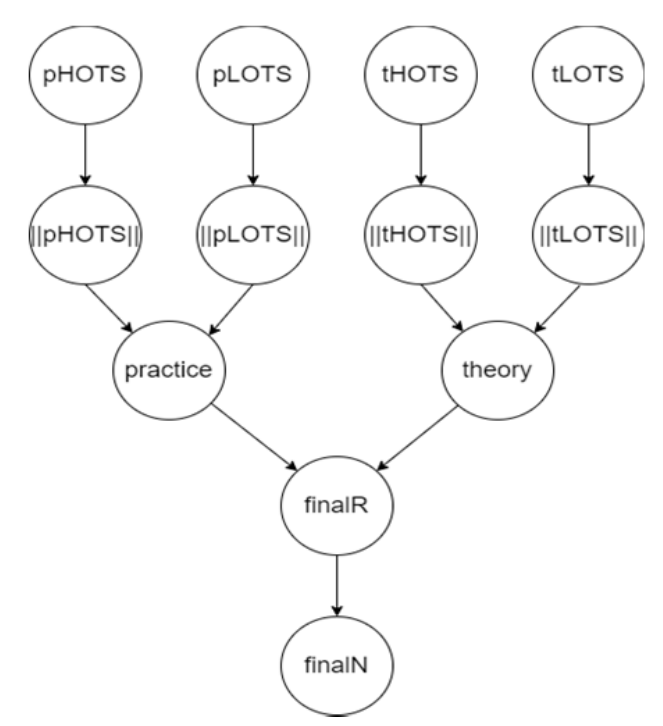

Figure 2.a) Variant 1: Theoretical and practical hierarchical multicomponent assessment based on Model 1

assessor's judgment. In the liberal approach, the scores from the primary components are reduced directly to the interval $[2,6]$ :

$$
\begin{aligned}
& \text { (4.1) } \| \text { tLOTS } \|=2+4 \frac{\text { tLOTS }}{30} \\
& \text { (4.2) } \| t \text { tHOTS } \|=2+4 \frac{t \text { tHOTS }}{15} \\
& \text { (4.3) }\|p L O T S\|=2+4 \frac{p \text { LOTS }}{15} \\
& \text { (4.4) \|pHOTS } \|=\text { pHOTS. }
\end{aligned}
$$

In the strict evaluation approach, the scores are initially displayed in the interval $[0,6]$. Assessments under 2,5 are rounded to 2. Accordingly, the membership functions for the second level components are:

$$
\begin{aligned}
& \text { (5.1.1) }\|t L O T S\|=\left\{\begin{array}{c}
2,6 \frac{\text { tLOTS }}{30}<2.5 \\
6 \frac{\text { tLOTS }}{30}, 6 \frac{\text { tLOTS }}{30} \geq 2.5
\end{array}\right. \\
& \text { (5.2.1) } \| \text { tHOTS } \|=\left\{\begin{array}{r}
2,6 \frac{\text { tHOTS }}{15}<2.5 \\
6 \frac{\text { tHOTS }}{15}, 6 \frac{\text { tHOTS }}{15} \geq 2.5
\end{array}\right. \\
& \text { (5.3.1) }\|p L O T S\|=\left\{\begin{array}{r}
2,6 \frac{\text { pLOTS }}{15}<2.5 \\
6 \frac{p L O T S}{15}, 6 \frac{p L O T S}{15} \geq 2.5
\end{array}\right.
\end{aligned}
$$$$
\text { (5.4) } \| \text { pHOTS } \|=\text { pHOTS. }
$$

Presented with IF-THEN logic, the formulas look like this:

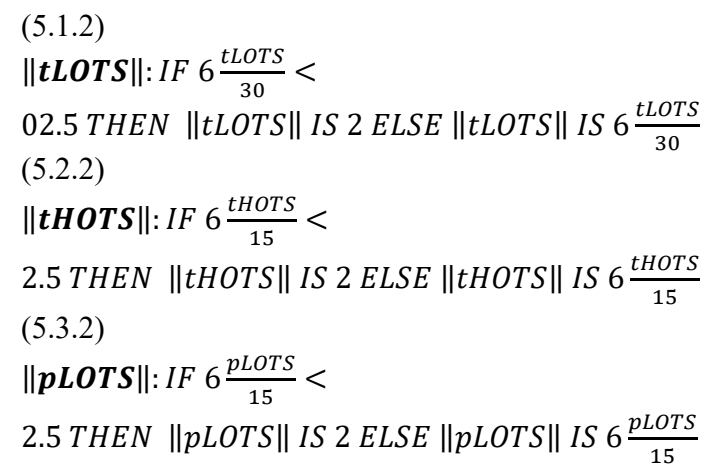

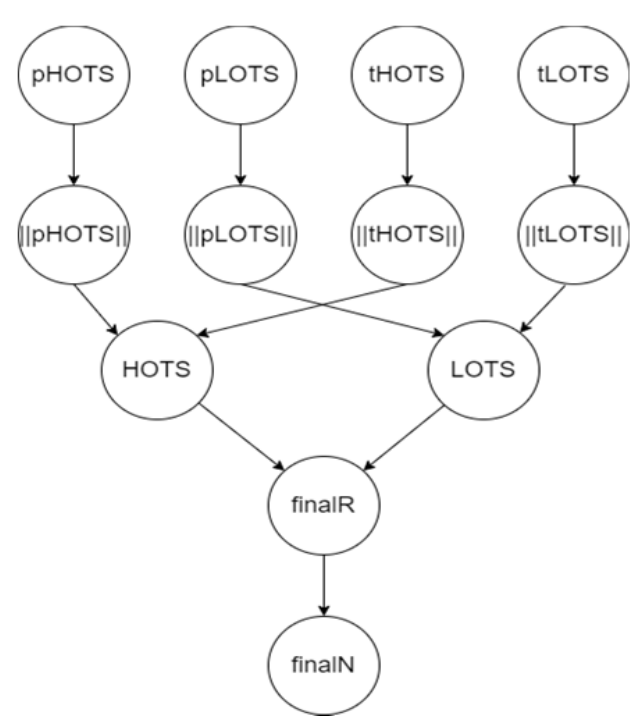

Figure 2.b) Variant 2: HOTS-LOTS hierarchical multicomponent assessment based on Model 1

More complex fuzzy logic needs to be used in the membership functions for the main components theory and practice. The arithmetic mean values of the sub-components are not always a good solution and therefore a subjective assessment is made by the assessor. There are many specific cases that need to be formalized to describe the assessor's logic. Some of the considerations used in our assessment to evaluate practice components are as follows:

- If pHOTS or pLOTS is 2 , the assessment of the practice component is no more than 3 even if the other sub-component has an assessment of 6 .

- If the assessment pHOTS is higher while pLOTS is low, then suspicions arise of dishonest behavior of the student in solving the task that forms the pHOTS assessment. The assessment should logically be lower than the arithmetic mean. This happens most often when pHOTS is close to 6 , and pLOTS is close to 3 .

- If pLOTS is high and pHOTS is low, then the knowledge demonstrated during the test ensures that the student has the necessary basis for future development in the discipline taught. Although he did not perform well in solving the task, the student deserves an incentive within reasonable limits. Of course, these limits must be preformalized with precise numerical values and rules.

Similar considerations apply to theoretical knowledge. As the tests forming tHOTS and tLOTS are usually performed in a controlled environment, dishonest student behavior is not common. Moreover, the high grades tHOTS are normally tolerated even with a partial lack of theoretical knowledge in tLOTS.

The specific rules we use to assess practice and theory in Variant 1 for theoretical and practical hierarchical multicomponent evaluation are as follows: 
(6.1) (practice $(\|p L O T S\|,\|p H O T S\|)$ :

IF $\frac{\|p H O T S\|+\|p L O T S\|}{2}<2.5$ THEN practice IS 2

ELSE IF $\|$ pLOTS $\|=2$ OR $\|$ pHOTS $\|=2$ THEN practice IS 3

ELSE IF (\|pHOTS\|

$-\|p L O T S\|)$

$\geq 2$

THEN practice IS $\left(\frac{\|p H O T S\|+\|p L O T S\|}{2}\right.$

$-0.5)$

ELSE practice IS $\left(\frac{\|p H O T S\|+\|p L O T S\|}{2}\right)$

(6.2) theory $(\|$ tLOTS $\|$,$\| tHOTS \|)$ :

IF $\frac{\| \text { tHOTS }\|+\| \text { tLOTS } \|}{2}<2.5$ THEN theory IS 2

ELSE IF $(\| t$ HOTS $\|-\|$ LLOTS $\|) \geq 2$

THEN theory IS $\left(\frac{\|t H O T S\|+\|t L O T S\|}{2}+0.3\right)$

ELSE theory IS $\left(\frac{\|t H O T S\|+\|t L O T S\|}{2}\right)$

To determine the final assessment in the Variant 1 presented, we have chosen to put equal weight to theory and practice and we have formulated the rules as follows:

(7) finalR(theory, practice):

IF $\frac{\text { theory }+ \text { practice }}{2}<2.5$ THEN finalR IS 2

ELSE IF theory $=2$ OR practice $=2$ THEN finalR IS 3

ELSE finalR IS $\left(\frac{\text { theory }+ \text { practice }}{2}\right)$

The final assessment finalN is defined by rounding finalR to the nearest integer:

(8) $\boldsymbol{f i n a l N}($ finalR $)=R O U N D($ final $R)$

Determining the assessments HOTS and LOTS in Variant 2 for HOTS-LOTS hierarchical multicomponent evaluation are also subject to similar considerations and can be customized according to the views of the particular assessor.

The evaluation formulas for the practice and theory components - (6.1) and (6.2) are compliant with Model 1, therefore, only components from the previous level are involved in them. In certain borderline cases it is suitable to use higher-level subcomponents, respectively Model 2 . The reason for this type of evaluation could be the following reasoning: The assessment for theory is 2 if the arithmetic mean value of the normalized assessments is lower than 2.5 and the pLOTS scores are less than 8 (in the strict assessment approach):

$$
\begin{gathered}
\text { IF } \frac{\|p H O T S\|+\|p L O T S\|}{2}<2.5 \text { AND pLOTS } \\
<8 \text { THEN practice IS } 2
\end{gathered}
$$

In our experiments on forming assessments according to different rules, we achieved the best approximation to the assessor's logic in the combination "Model 2 - Variant 1 - Strict approach".

Creating a workable solution at a certain time for a certain discipline, course and assessors does not guarantee its effective applicability in the future, for other courses or other assessors. Initial settings and several automatically obtained correct estimates do not release the assessor from the responsibility for monitoring and control of the subsequent results. Unforeseen or inaccurate rules could distort the assessor's logic in certain traditional or borderline cases.

The advantage of using fuzzy logic in evaluation is the ability to achieve accurate automated assessment according to the subjective assessor's logic. The main disadvantage is the difficulty for non-specialists to formalize the rules for forming the assessment. Another disadvantage compared to the artificial intelligence methods studied by us is the need for specific work in determining the rules and checking their correctness.

The main conclusion of the conducted study is that the use of fuzzy logic ensures the receiving of correct grades, with correctly written rules, while the use of artificial intelligence methods requires a sufficiently large number of appropriate input-output samples for training. The creation of input-output samples in artificial intelligence methods is part of the natural evaluation process, while determining formal logic requires mathematical and algorithmic thinking. It should be noted here that all automated approaches require subsequent monitoring and control by the teacher of the automatically formed assessments.

\section{Conclusion}

Complex and objective evaluation of students' knowledge and skills is an important element of the educational process both for students and teachers. On the one hand, the evaluation enables teachers to monitor the progress of learners, to assess whether the learning objectives are met, to estimate the learners' needs, to evaluate the effectiveness of teaching and learning. On the other hand, the evaluation enables learners to identify their strengths 
and weaknesses, compare themselves with their peers, and take responsibility for their own progress. It is for these reasons that teachers need to focus on the assessment process and look for working evaluation models appropriate for their students and the cources they teach.

The theory of fuzzy sets provides a tool for formalizing the assessor's subjective logic in mathematical and software models. The proposed models for hierarchical multicomponent evaluation of students aim at comprehensive evaluation of different thinking skills of high and low order, theoretical knowledge and practical skills, etc. They allow the use of fuzzy logic and can be adapted for use in assessing different disciplines in different age groups. A specific case of using the proposed models has been successfully experimented with students studying in the discipline "Internet Programming".

\section{Acknowledgements}

The work is funded by the SP19-FMI-004 project at the Research Fund of the University of Plovdiv "Paisii Hilendarski".

\section{References}

[1]. Furst, M. D., Hill, E. J., Krathwohl, W. H., Bloom, D. R., \& Engelhart, B. S. (1956). Taxonomy of educational objectives: The classification of educational goals. Handbook I: Cognitive Domain. New York: David McKay Company.

[2]. Tuzharov, Hr. (2009). E-learning and trends in higher education, Sofia, Bulgaria: Asenevtsi.

[3]. Brookhart, S. M. (2010). How to assess higher-order thinking skills in your classroom. ASCD.

[4]. Vasileva, M. (2008). Fuzzy sets. Theory and practice, Shumen, Bulgaria: NVU Vasil Levski.

[5]. Mardani, A., Jusoh, A., Nor, K., Khalifah, Z., Zakwan, N., \& Valipour, A. (2015). Multiple criteria decision-making techniques and their applications-a review of the literature from 2000 to 2014. Economic Research-Ekonomska Istraživanja, 28(1), 516-571.

[6]. Peneva, V., \& Popchev, I. (2009). Multicriteria Decision Making by Fuzzy Relations and Weighting Functions for the Criteria. Cybernetics and Information Technologies, 9(4), 58-71.
[7]. DeLong, C., Radcliffe, P., \& Gorny, L. (2007). Recruiting for retention: Using data mining and machine learning to leverage the admissions process for improved freshman retention. In Proc. of the Nat. Symposium on Student Retention.

[8]. Kabakchieva, D. (2013). Predicting student performance by using data mining methods for classification. Cybernetics and information technologies, 13(1), 61-72.

[9]. Nandeshwar, A., \& Chaudhari, S. (2009). Enrollment prediction models using data mining.

Retrieved from: http://nandeshwar.info/wpcontent/uploads/2008/11/DMWVU_Project.pdf [accessed: 10 May 2020].

[10]. Radeva, I. (2013). Multi-Criteria Models for Clusters Design. Cybernetics and Information Technologies, 13(1), 18-33.

[11]. Borissova, D., \& Keremedchiev, D. (2019). Group decision making in evaluation and ranking of students by extended simple multi-attribute rating technique. Cybernetics and Information Technologies, 19(3), 45-56.

[12]. Langova-Orozova, D., Aladjov, H., \& Atanasov, K. (2001). Generalized Net Fof Machine Learning With Current Estimations. Advanced Studies in Contemporary Mathematics, Ku-Duk Press, 3(02), 61-76.

[13]. Orozova, D. (2007). Generalized Net Model of Tutoring System. Intuitionistic Fuzzy Sets and Generalized Nets, 5, 25-34.

[14]. Sotirova, E., Atanassov, K., Shannon, A., Kim, T., Krawczak, M., Melo-Pinto, P., \& Riečan, B. (2016, September). Intuitionistic fuzzy evaluations for analysis of a student's knowledge of mathematics in university e-learning courses. In 2016 IEEE 8th International Conference on Intelligent Systems (IS) (pp. 535-537). IEEE.

DOI: $10.1109 /$ IS.2016.7737474.

[15]. Shannon, A., Sotirova, E., Petrounias, I., Atanassov, K., Krawczak, M., Melo-Pinto, P., \& Kim, T. (2006, December). Generalized net model of lecturers' evaluation of student work with intuitionistic fuzzy estimations. In Second International Workshop on Intuitionistic Fuzzy Sets, Banska Bystrica, Slovakia (Vol. 3, pp. 22-28).

[16]. Hadzhikolev, E., Yotov, K., Trankov, M., \& Hadzhikoleva, S. (2019). Use of Neural Networks in Assessing Knowledge and Skills of University Students. In ICERI2019 Proceedings (pp. 7474-7484). IATED. DOI: 10.21125/iceri.2019.1787. 